K. Hayasida and M. Nakatani

Nagoya Math. J.

Vol. 157 (2000), 177-209

\title{
ON THE DIRICHLET PROBLEM OF PRESCRIBED MEAN CURVATURE EQUATIONS WITHOUT H-CONVEXITY CONDITION
}

\author{
KAZUYA HAYASIDA AND MASAO NAKATANI
}

\begin{abstract}
The Dirichlet problem of prescribed mean curvature equations is well posed, if the boundery is H-convex. In this article we eliminate the Hconvexity condition from a portion $\Gamma$ of the boundary and prove the existence theorem, where the boundary condition is satisfied on $\Gamma$ in the weak sense.
\end{abstract}

\section{$\S 1$. Introduction}

Let $\Omega$ be a bounded domain in $R^{n}, n \geqq 2$, with its boundary $\partial \Omega$. We denote by $\left(x_{1}, \ldots, x_{n}\right)$ the coordinates in $R^{n}$ and write $D=\left(D_{1}, \ldots, D_{n}\right)$, where $D_{i}=\partial / \partial x_{i}$.

We consider the Dirichlet problem

$$
D \cdot\left(\frac{D u}{\sqrt{1+|D u|^{2}}}\right)=n H \quad \text { in } \Omega
$$

with

$$
u=\phi \quad \text { on } \partial \Omega,
$$

which was studied by many authors. The equation (1.1) is called by the prescribed mean curvature equation.

Let $|\Omega|$ and $\omega_{n}$ be two volumes of $\Omega$ and the unit ball in $R^{n}$, respectively. Throughout this article we assume

$$
\sup _{\Omega}|H|<\frac{1}{n}\left(\frac{\omega_{n}}{|\Omega|}\right)^{1 / n}
$$

which may be replaced in some weaker conditions. That is, (1.3) means that

$$
\int_{\Omega}|H|^{n} d x<\omega_{n}
$$

Received August 26, 1993.

Revised March 19, 1999. 
and for some $\varepsilon_{0}>0$

$$
\left|\int_{\Omega} H \eta d x\right| \leq \frac{1-\varepsilon_{0}}{n} \int_{\Omega}|D \eta| d x, \quad \eta \in C_{0}^{1}(\Omega) .
$$

But we impose (1.3) on this article for the sake of simplicity.

Serrin [17] solved first the Dirichlet problem (1.1) with (1.2). His result is as follows: Suppose that $\partial \Omega \in C^{2, \alpha}, \phi \in C^{2, \alpha}(\bar{\Omega})$ for some $\alpha$ with $0<$ $\alpha<1$, and $H \in C^{1}(\bar{\Omega})$. If (1.3) and

$$
\frac{n}{n-1}|H| \leq \Lambda
$$

are assumed on $\partial \Omega$, then the problem (1.1) with (1.2) is uniquely solvable for $u \in C^{2, \alpha}(\bar{\Omega})$, where $\Lambda$ is the boundary mean curvature of $\Omega$.

The condition (1.6) is called by $H$-convexity. Afterward Serrin's result was extended to the generalized mean curvature equation of higher order by Ivochkina [6], whose study is closely related to the fully nonlinear elliptic equation. Recently, Gregori [5] studied the relation between BV solutions and viscosity solutions for (1.1).

By weakening the above assumptions except for (1.6), many authors solved the problem (1.1) with (1.2), where the required solutions $u$ are in $C^{2}(\Omega) \cap C(\bar{\Omega})$ (see e.g., [1], [2], [4], [18], [20]). The starting point is in two ways. One is to estimate the generalized BV solution (see e.g., [4]). Another is to estimate the approximating solution of each perturbed uniformly elliptic equation (see e.g., [18]). In either case it needs to construct the barrier functions. Further there are a few papers which prove $u-\phi \in W_{0}^{1,1}(\Omega)$ (see e.g., [15], [20]). Their method is also to construct the barrier function. So, it is difficult to drop the condition (1.6).

Suppose that (1.6) is not assumed. Let $H=0$, namely (1.1) be the minimal surface equation. Then the problem (1.1) with (1.2) is solvable, if $\phi$ is small concerning some norm (see [14], [22]). When $H \neq 0$, there is the result of Schulz and Williams [16]. Lancaster [12] showed the nonexistence of solutions for some domain having a reentrant corner. Recently, Jin and Lancaster [8] investigated the behavior near a reentrant corner of a solution to a quasilinear elliptic equation in a two dimensional domain. And Tersenov [21] proved the existence of $C^{2, \alpha}(\bar{\Omega})$ solutions of the Dirichlet problem with zero boundary conditions for quasilinear elliptic equations in some non convex domains $\Omega$. In [21] the condition on $\Omega$ is complicated to be stated. On the other hand, when $H=0$, Jenkins and Serrin [7] showed 
previously that a necessary and sufficient condition on $\partial \Omega$ for the solvability of the Dirichlet problem (1.1) with (1.2) for arbitrary continuous $\phi$ is that $\Lambda \geqq 0$ everywhere. Afterward Williams [23] constructed a $C^{\infty}$-domain in $R^{2}$ and a $C^{\infty}$-function $\phi$ such that the limit of the generalized solution at a point on $\partial \Omega$ from inward does not exist.

We consider the case of $H=0$. Let $u$ be the generalized BV solution. The assumption (1.6) guarantees that $u=\phi$ on $\partial \Omega$. We suppose that $\phi \in$ $C^{0,1}(\partial \Omega)$ and $\partial \Omega$ is of class $C^{4}$. Let $A=\{x \in \partial \Omega \mid \Lambda(x)<0, u(x) \neq \phi(x)\}$. Then by Lau and Lin [13] it was proved that $u$ is Hölder continuous near $A$ with exponent exactly $1 / 2$, and the trace of $u$ over $A$ is regular according to the regularity of $\partial \Omega$. More precise results were obtained by Korevaar and Simon [9] and Simon [19].

In this article our aim is as follows: Let $\Gamma$ be a portion of $\partial \Omega$, where (1.6) is not assumed. Instead we assume that $\Gamma$ is transformed into a hyperplane by an orthogonal coordinates mapping (see the Definition in the beginning of Section 2). Then we shall show that there exists a solution $u$ of the Dirichlet problem (1.1) with (1.2) such that for some $\alpha>0,(u-\phi) /\left(1+|D u|^{2}\right)^{\alpha}$ belongs to $W^{1,2}$ near $\Gamma$ and its trace over $\Gamma$ equals 0 (see Theorem 1 ). This statement means that $u=\phi$ on $\Gamma$ in the weak sense. In fact the equality $u=\phi$ on $\Gamma$, is equivalent to that the trace of $(u-\phi) /\left(1+|D u|^{2}\right)^{\alpha}$ vanishes there, if $u$ is smooth. Next we shall give a sufficient condition in order that $u-\phi \in W^{1,1}(\Omega)$ and its trace vanishes on $\Gamma$ (see Theorem 2).

There is the result of Ladyzhenskaya and Ural'ceva [10] and [11] concerning the local interior estimate of approximating solutions. In [11] particularly, the gradient bound in the interior domain was proved. In this article, by using the method in [10], we prepare some boundary estimates in order to prove our theorems.

\section{$\S$ 2. Result}

From now on, let $\Omega$ be a bounded domain, and $\partial \Omega$ be locally Lipschitzcontinuous. We denote by $B_{\delta}(P)$ the open ball in $R^{n}$ with its center $P$ and with its radius $\delta$. We set the following

Definition. We say that $P \in \partial \Omega$ has property (A), if the following holds:

There exist a positive number $\delta$ and an one-to-one mapping $\Phi$

$$
\Phi: B_{\delta}(P) \ni\left(x_{1}, \ldots, x_{n}\right) \longmapsto\left(\xi_{1}, \ldots, \xi_{n}\right) \in R^{n}
$$


satisfying

(I) $\Phi$ and $\Phi^{-1}$ are both of class $C^{3}$ such that

$$
\frac{D\left(\xi_{1}, \ldots, \xi_{n}\right)}{D\left(x_{1}, \ldots, x_{n}\right)}>0 \quad \text { in } B_{\delta}(P) .
$$

(II) $\Phi(P)=O, \Phi\left(B_{\delta}(P) \cap \Omega\right) \subset\left\{\xi_{n}>0\right\}$ and

$$
\Phi\left(B_{\delta}(P) \cap \partial \Omega\right) \subset\left\{\xi_{n}=0\right\}
$$

(III) $D_{x} \xi_{i} \cdot D_{x} \xi_{j}=0$ in $B_{\delta}(P)$, if $i \neq j$.

We denote by $\mathbf{n}$ and $\tau$ the inward normal vector and the tangent vector at $\partial \Omega \cap B_{\delta / 2}(P)$, respectively. Then from the above (III) we have

$$
\frac{\partial}{\partial \mathbf{n}}=a_{n} D_{\xi_{n}} \quad \text { and } \quad \frac{\partial}{\partial \tau}=\sum_{i=1}^{n-1} a_{i} D_{\xi_{i}}
$$

where $a_{i}$ are $C^{2}$ functions such that $a_{n}>0$ and $\sum_{i=1}^{n-1}\left|a_{i}\right| \neq 0$ on $\partial \Omega \cap$ $B_{\delta / 2}(P)$. Let $1 \leq p<\infty$ and $\Gamma$ be an open set on $\partial \Omega$. We define

$$
\begin{gathered}
W_{0}^{1, p}(\Omega ; \Gamma)=\left\{u \mid u \in W^{1, p}\left(\Omega^{\prime}\right) \text { and the trace of } u \text { over } \partial \Omega^{\prime} \cap \Gamma\right. \\
\text { vanishes for any subdomain } \Omega^{\prime} \text { of } \Omega \text { such that } \\
\left.\bar{\Omega}^{\prime} \cap(\partial \Omega-\Gamma)=\phi \text { and } \overline{\partial \Omega^{\prime} \cap \Gamma} \subset \Gamma\right\} .
\end{gathered}
$$

Throughout this article we set the following assumptions:

We take two relatively open subsets $\Gamma_{1}$ and $\Gamma_{2}$ of $\partial \Omega$, where $\Gamma_{1}$ is of class $C^{3}$ and each point of $\Gamma_{1}$ has property $(\mathrm{A})$. It is not assumed that $\partial \Omega=\Gamma_{1} \cup \Gamma_{2}$. Let $H$ be a function in $C^{0,1}(\bar{\Omega})$ satisfying (1.3). Let $\phi$ be a function in $C^{2,1}\left(\Omega \cup \bar{\Gamma}_{1}\right) \cap C^{0}(\bar{\Omega}) \cap W^{1,1}(\Omega)$. Further (1.6) is imposed only on $\Gamma_{2}$, namely it is not assumed on $\Gamma_{1}$.

Then we solve the Dirichlet problem (1.1) with (1.2). Our first aim is to prove

THEOREM 1. There exists a solution $u \in C^{2}(\Omega) \cap C^{0}\left(\Omega \cup \Gamma_{2}\right) \cap W^{1,1}(\Omega)$ of (1.1) such that

$$
u=\phi \text { on } \Gamma_{2}, \quad \text { and } \quad \frac{u-\phi}{\left(1+|D u|^{2}\right)^{5 / 4}} \in W_{0}^{1,2}\left(\Omega ; \Gamma_{1}\right)
$$


As stated at the end of the previous section, the last relation in Theorem 1 is regarded as $u=\phi$ on $\Gamma_{1}$, in the weak sense. We take a sequence of domains $\left\{\Omega_{j}\right\}$ as follows: Each $\partial \Omega_{j}$ is of class $C^{3}$ and $\Omega_{1} \subset \Omega_{2} \subset \cdots \subset \Omega_{j} \rightarrow$ $\Omega(j \rightarrow \infty)$. Further for any compact set $K$ in $\Gamma_{1}$, it holds that $\partial \Omega_{j_{0}} \supset K$ for some $j_{0}$. Obviously there is a sequence $\left\{\phi_{j}\right\} \subset C^{3}(\bar{\Omega})$ satisfying

$$
\phi_{j} \rightarrow \phi \quad \text { in } C^{2,1}\left(\Omega \cup \bar{\Gamma}_{1}\right) \cap C^{0}(\bar{\Omega}) \cap W^{1,1}(\Omega)(j \rightarrow \infty) .
$$

We take a positive sequence $\left\{\varepsilon_{j}\right\}$ such that $\varepsilon_{j} \rightarrow 0(j \rightarrow \infty)$ and $\varlimsup_{j \rightarrow \infty}\left(\varepsilon_{j} \int_{\Omega}\left|D \phi_{j}\right|^{2} d x\right)<\infty$. It is known that for each $j$ there is a solution $u_{j} \in C^{2}\left(\bar{\Omega}_{j}\right)$ of

$$
\left\{\begin{aligned}
\varepsilon_{j} \Delta u_{j}+D \cdot\left(\frac{D u_{j}}{\sqrt{1+\left|D u_{j}\right|^{2}}}\right) & =n H \quad \text { in } \Omega_{j} \\
u_{j} & =\phi_{j} \quad \text { on } \partial \Omega_{j}
\end{aligned}\right.
$$

(see [3]).

According to the result of Simon [18], there is a subsequence $\left\{u_{\nu}\right\}$ of $\left\{u_{j}\right\}$ and a function $u \in C^{2}(\Omega)$ such that for any compact subset $K$ of $\Omega$

$$
D^{\alpha} u_{\nu} \rightrightarrows D^{\alpha} u \quad \text { in } K(\nu \rightarrow \infty), \quad|\alpha| \leq 2,
$$

$u \in C^{0}\left(\Omega \cup \Gamma_{2}\right), u=\phi$ on $\Gamma_{2}$ and (1.1) holds. The reason for its validity is due to $(1.6)$.

In [18] the following equation was considerd in place of that in (2.1):

$$
\varepsilon_{j} \Delta u_{j}+\left(1-\varepsilon_{j}\right) D \cdot\left(\frac{D u_{j}}{\sqrt{1+\left|D u_{j}\right|^{2}}}\right)=n H .
$$

But the situation is quite parallel.

Next we have

TheOrem 2. Assume that $\partial u_{j} / \partial \mathbf{n} \geqq 0$ on $\Gamma_{1} \cap \partial \Omega_{j}$, for each $u_{j}$. Then there is a positive constant $d_{0}$ depending only on the shape of $\Gamma_{1}$ such that if $H \geqq d_{0}$ on $\Gamma_{1}$, the equation (1.1) is solvable for $u \in C^{2}(\Omega) \cap C^{0}\left(\Omega \cup \Gamma_{2}\right)$ satisfying

$$
u=\phi \text { on } \Gamma_{2}, \quad u-\phi \in W^{1,1}(\Omega) \cap W_{0}^{1,1}\left(\Omega ; \Gamma_{1}\right)
$$

and

$$
\frac{u-\phi}{\left(1+|D u|^{2}\right)^{1 / 4}} \in W_{0}^{1,2}\left(\Omega ; \Gamma_{1}\right)
$$


The constant $d_{0}$ will be concretely given in the proof of Proposition 4.3 (see (4.27)). Though it is almost impossible to verify the assumption in the above theorem, we give two examples to show that Theorem 2 is not meaningless. For this sake we prepare the following.

Let $D$ be a bounded domain in $R^{n}$ and $\tilde{H}(x)$ be a bounded function in $D$. For $\varepsilon>0$ we define the operator $Q^{\varepsilon}$ :

$$
\begin{aligned}
Q^{\varepsilon} u=( & \left.\varepsilon \sqrt{1+|D u|^{2}}+1\right)\left(1+|D u|^{2}\right) \Delta u \\
& -D_{i} u \cdot D_{j} u \cdot D_{i} D_{j} u-n \tilde{H}(x)\left(1+|D u|^{2}\right)^{3 / 2}
\end{aligned}
$$

Then the following assertion holds, which is due to Theorem 10.1 in [3].

Suppose that $u, v \in C^{2}(D) \cap C^{1}(\bar{D})$ and $Q^{\varepsilon} v \geqq Q^{\varepsilon} u$ in $D$. Then $v \leqq u$ in $D$, if $v \leqq u$ on $\partial D$.

Here we assume that $\partial D$ is of class $C^{1}$. Let $\Gamma$ be an open subset of $\partial D$. Let $\tilde{\phi} \in C^{2,1}(\bar{D})$ and $\tilde{H} \in C^{0,1}(\bar{D})$. Then we have

Proposition 2.1. Suppose that $u$ is a function in $C^{2}(D) \cap C^{1}(\bar{D})$ satisfying

$$
Q^{\varepsilon} u=0 \text { in } D \quad \text { and } u=\tilde{\phi} \text { on } \partial D
$$

Then $\partial u / \partial \mathbf{n} \geqq 0$ on $\Gamma$, if there is a function $v \in C^{2}(D) \cap C^{1}(\bar{D})$ such that

$$
\begin{aligned}
& Q^{\varepsilon} v \geqq 0 \text { in } D, \quad v \leqq \tilde{\phi} \text { on } \partial D, \\
& v=\tilde{\tilde{\phi}} \text { on } \Gamma \quad \text { and } \quad \partial v / \partial \mathbf{n} \geqq 0 \text { on } \Gamma \text {. }
\end{aligned}
$$

Proof. By the previous assertion we see that $v \leqq u$ in $\bar{D}$. Since $v=u$ on $\Gamma$, it holds that $\partial v / \partial \mathbf{n} \leqq \partial u / \partial \mathbf{n}$ on $\Gamma$. This completes the proof.

We give the following two examples satisfying the assumptions in Theorem 2 .

ExAmPLE 1. Let $\Gamma_{1}$ be the arc defined in the example given at the end of Section 4 , where we put $R=1$. Let $\Omega$ be a bounded domain such that $\Omega$ lies up $\Gamma_{1}$ and $\partial \Omega \supset \Gamma_{1}$ (see Figure 1 ).

Let $\Gamma_{2}=\partial \Omega-\bar{\Gamma}_{1}$. We assume

$$
1<\sqrt{\frac{\pi}{|\Omega|}} .
$$

As stated in this section, we take the approximating sequence $\left\{\Omega_{j}\right\}$ of $\Omega$. 


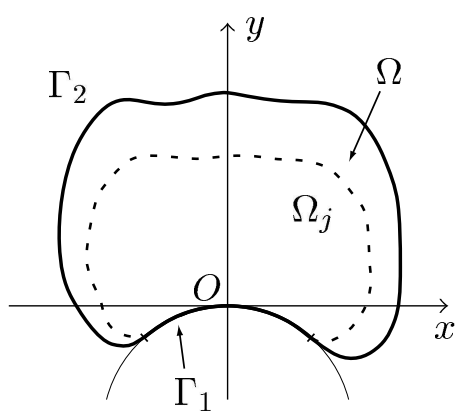

Figure 1.

Let $H$ be a positive function in $C^{0,1}(\bar{\Omega})$ such that

$$
\frac{1}{2} \leqq H<\frac{1}{2} \sqrt{\frac{\omega_{2}}{|\Omega|}} .
$$

From (2.3) it is possible to take such a function $H$. Retaking $\Gamma_{2}$, we may assume that (1.6) holds there.

We define two positive numbers $d_{1}$ and $d_{2}$ as follows:

$$
d_{1}=-\inf \{y \mid(x, y) \in \Omega\}, \quad d_{2}=\sup \{y \mid(x, y) \in \Omega\}
$$

Taking two real numbers $A$ and $B$, we set

$$
v(x, y)=A\left(y+d_{1}\right)^{2}+B
$$

Let us impose the following assumptions on $A$ and $d_{1}+d_{2}$ :

$$
A \geqq\left(\sup _{\Omega}|H|\right)\left(1+4 A^{2}\left(d_{1}+d_{2}\right)^{2}\right)^{3 / 2} .
$$

The two relations (2.4) and (2.5) are not contradictory each other.

Let $\phi$ be a function such that $\phi \geqq v$ in $\Omega$ and $\phi=v$ on $\Gamma_{1}$. Under the above conditions we set $\varepsilon=\varepsilon_{j}, D=\Omega_{j}, \Gamma=\Gamma_{1} \cap \partial \Omega_{j}, \tilde{H}=H$ and $\tilde{\phi}=\phi$ in Proposition 2.1. From (2.4) the condition (1.3) on $H$ is satisfied. Let $u_{j}$ be the solution satisfying

$$
Q^{\varepsilon_{j}} u_{j}=0 \text { in } \Omega_{j} \text { and } u_{j}=\phi \text { on } \partial \Omega_{j}
$$

Previously we may assume

$$
\partial v / \partial \mathbf{n} \geqq 0 \quad \text { on } \Gamma_{1} .
$$


From (2.5) we see that $Q^{\varepsilon_{j}} v \geqq 0$ in $\Omega$. Therefore it follows from Proposition 2.1 that

$$
\partial u_{j} / \partial \mathbf{n} \geqq 0 \quad \text { on } \Gamma_{1} \cap \partial \Omega_{j} .
$$

In this case the positive constant $d_{0}$ in Theorem 2 will be calculated exactly in the example given at the end of Section 4 . That is, we can take $d_{0}=1 / 2$, which is independent of $d_{1}+d_{2}$. The conditions (2.4) with (2.6), mean that this example satisfies the assumptions in Theorem 2.

EXAmPle 2. Next let $\Omega$ be the annular domain such as

$$
\Omega=\left\{(x, y) \mid 0<R_{1}<r<R_{2}\right\}, \quad r=\sqrt{x^{2}+y^{2}} .
$$

We set $\Gamma_{1}=\left\{r=R_{1}\right\}$ and $\Gamma_{2}=\left\{r=R_{2}\right\}$ (see Figure 2). We put $\Omega_{j}=\Omega$

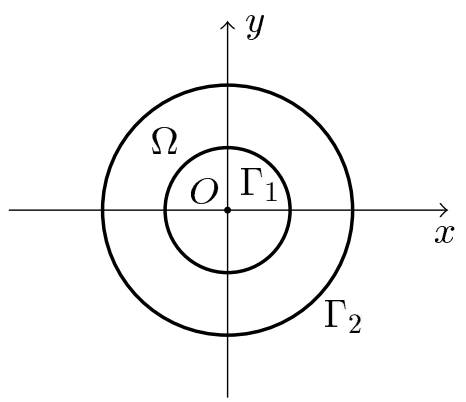

Figure 2.

for all $j$. Let $H$ be a positive function in $C^{0,1}(\bar{\Omega})$ satisfying (1.3), where $|\Omega|=\pi\left(R_{2}^{2}-R_{1}^{2}\right)$. It is known that the boundary mean curvature $\Lambda$ equals $1 / R_{2}$ at each point on $\Gamma_{2}$.

We assume

$$
H \geqq \frac{1}{2 R_{1}} \text { on } \Gamma_{1} \quad \text { and } \quad H \leqq \frac{1}{2 R_{2}} \text { on } \Gamma_{2} .
$$

Then (1.6) is satisfied on $\Gamma_{2}$. Taking two real numbers $A$ and $B$, we define

$$
v(x, y)=A r^{2}+B
$$

and assume that

$$
2 A+4 A^{3} r^{2}>H\left(1+4 A^{2} r^{2}\right)^{3 / 2} \text { for } R_{1} \leqq r \leqq R_{2} .
$$


Then it is seen that $Q^{\varepsilon_{j}} v \geqq 0$ in $\Omega$. And obviously, $\partial v / \partial \mathbf{n} \geqq 0$ on $\Gamma_{1}$. Let $\phi$ be a function such that $\phi \geqq v$ in $\Omega$ and $\phi=v$ on $\Gamma_{1}$. We take $u_{j}$ in such a way that

$$
Q^{\varepsilon_{j}} u_{j}=0 \text { in } \Omega \text { and } u_{j}=\phi \text { on } \partial \Omega .
$$

Applying Proposition 2.1, we have

$$
\partial u_{j} / \partial \mathbf{n} \geqq 0 \quad \text { on } \Gamma_{1} .
$$

Similarly as in Example 1, we can take $d_{0}=1 / 2 R_{1}$. The following inequality holds:

$$
2 t+4 t^{3}>\frac{1}{2}\left(1+4 t^{2}\right)^{3 / 2} \text { for } t \geqq \frac{1}{2} .
$$

This means that we can take a function $H$ and four numbers $A, B, R_{1}$ and $R_{2}$ satisfying the above conditions.

\section{$\S 3$. Preliminaries}

In this section we prepare some known results for solutions $u_{j}$ in (2.1). We denote by $(,)_{j}$ the $L^{2}\left(\Omega_{j}\right)$-inner product. Setting $v_{j}=u_{j}-\phi_{j}$, we multiply (2.1) with $v_{j}$. Then by integration by parts, we have from (1.5)

$$
\begin{aligned}
& \varepsilon_{j}\left(1,\left|D v_{j}\right|^{2}\right)_{j}+\left(\frac{D u_{j}}{\sqrt{1+\left|D u_{j}\right|^{2}}}, D u_{j}\right)_{j} \\
& \quad \leqq-\varepsilon_{j}\left(D v_{j}, D \phi_{j}\right)_{j}+\left(\frac{D u_{j}}{\sqrt{1+\left|D u_{j}\right|^{2}}}, D \phi_{j}\right)_{j}+\left(1-\varepsilon_{0}\right)\left(1,\left|D v_{j}\right|\right)_{j} .
\end{aligned}
$$

We use the assumption on $\left\{\varepsilon_{j}\right\}$ and the inequality $|t|-1 \leqq t^{2} / \sqrt{1+t^{2}}$ $(t \in R)$. Then it follows that

$$
\varepsilon_{j}\left(1,\left|D u_{j}\right|^{2}\right)_{j}+\left(1,\left|D u_{j}\right|\right)_{j} \leqq C
$$

where $C$ depends on $H, \phi$ and $\Omega$, but not on $j$.

Next we verify the uniform boundedness of $u_{j}$ with respect to $j$. If $\varepsilon_{j}=0$, this is due to Chapter 10 in [3]. So, we can proceed in parallel with it. We rewrite the equation in (2.1) with

$$
\begin{aligned}
Q_{j} u_{j}=\varepsilon_{j} & \left(1+\left|D u_{j}\right|^{2}\right)^{3 / 2} \Delta u_{j}+\left(1+\left|D u_{j}\right|^{2}\right) \Delta u_{j} \\
& \quad-D_{k} u_{j} \cdot D_{h} u_{j} \cdot D_{k h} u_{j}-n H\left(1+\left|D u_{j}\right|^{2}\right)^{3 / 2}=0 .
\end{aligned}
$$


We put

$$
\begin{aligned}
p & =\left(p_{1}, \ldots, p_{n}\right), \\
a_{j}^{k h}(p) & =\varepsilon_{j}\left(1+|p|^{2}\right)^{3 / 2} \delta_{k h}+\left(1+|p|^{2}\right) \delta_{k h}-p_{k} p_{h}, \\
b(x, p) & =-n H(x)\left(1+|p|^{2}\right)^{3 / 2} \\
\mathfrak{D}_{j} & =\operatorname{det}\left[a_{j}^{k h}(p)\right] \text { and } \mathfrak{D}_{j}^{*}=\mathfrak{D}_{j}^{1 / n} .
\end{aligned}
$$

The equation (3.2) becomes $a_{j}^{k h}\left(D u_{j}\right) D_{k} D_{h} u_{j}+b\left(x, D u_{j}\right)=0$. In virtue of Theorem 10.5 in [3], the following assertion holds:

Let $g(p)$ be in $L_{l o c}^{n}\left(R^{n}\right)$ and $h(x)$ be in $L^{n}\left(\Omega_{j}\right)$ such that

$$
\frac{|b(x, p)|}{n \mathfrak{D}_{j}^{*}} \leqq \frac{h(x)}{g(p)} \quad \text { for }(x, p) \in \Omega_{j} \times R^{n}
$$

and

$$
\int_{\Omega_{j}} h^{n} d x \leqq \int_{R^{n}} g^{n} d p .
$$

Then the solution $u_{j}$ of (3.2) satisfies

$$
\sup _{\Omega_{j}}\left|u_{j}\right| \leqq \sup _{\partial \Omega_{j}}\left|u_{j}\right|+C_{0} d\left(\Omega_{j}\right)
$$

where $d\left(\Omega_{j}\right)$ is the diameter of $\Omega_{j}$ and $C_{0}$ depends only on $g$ and $h$.

In our case we easily see that

$$
\mathfrak{D}_{j}=\left(1+\varepsilon_{j}\left(1+|p|^{2}\right)^{3 / 2}\right)\left(1+|p|^{2}+\varepsilon_{j}\left(1+|p|^{2}\right)^{3 / 2}\right)^{n-1} .
$$

Hence $\mathfrak{D}_{j}^{*} \geqq\left(1+|p|^{2}\right)^{(n-1) / n}$ and

$$
\frac{|b(x, p)|}{n \mathfrak{D}_{j}^{*}} \leqq|H(x)|\left(1+|p|^{2}\right)^{-(n+2) /(2 n)} .
$$

It is enough to set

$$
h(x)=|H(x)| \quad \text { and } \quad g(p)=\left(1+|p|^{2}\right)^{-(n+2) /(2 n)} .
$$

Since

$$
\int_{R^{n}} g^{n} d p=\omega_{n}, \quad \int_{\Omega_{j}} h^{n} d x<\omega_{n} \quad(\text { from }(1.4))
$$


the assumptions on the above assertion are satisfied. We define the function $G(t), t \geqq 0$ with

$$
G^{-1}(t)=\int_{B_{t}(O)} g^{n} d p,
$$

where $B_{t}(O)=\{|p|<t\}$. Then $G$ is a function from $\left(0, \omega_{n}\right)$ onto $(0, \infty)$. The constant $C_{0}$ on (3.3) is given by $G\left(\int_{\Omega} h^{n} d x\right)$, which is referred to [3].

Let $u$ be the function in (2.2). We show that

$$
u \in W^{1,1}(\Omega) .
$$

Hereafter we denote by the same $\{\nu\}$ any subsequence of $\{j\}$. Let $\Omega^{\prime}$ be any fixed subdomain of $\Omega$ with $\bar{\Omega}^{\prime} \subset \Omega$. From (3.1), $\int_{\Omega^{\prime}}\left(\left|D u_{j}\right|^{1 / 2}\right)^{2} d x$ are uniformly bounded with respect to $j$. Hence

$$
\left|D u_{\nu}\right|^{1 / 2} \longrightarrow g \text { weakly in } L^{2}\left(\Omega^{\prime}\right) \text { as } \nu \rightarrow \infty
$$

Thus

$$
\int_{\Omega^{\prime}} g^{2} d x \leqq \lim _{\nu \rightarrow \infty} \int_{\Omega^{\prime}}\left|D u_{\nu}\right| d x
$$

The right-hand side is uniformly bounded with respect to $\Omega^{\prime}$ in virtue of (3.1). This means that $g \in L^{2}(\Omega)$. On the other hand $g=|D u|^{1 / 2}$ from (2.2). Hence (3.5) is correct.

\section{§4. Main estimate}

We suppose the assumptions in the beginning of Section 2. Let $u_{j}$ be the solution of (2.1). Let $P$ be any fixed point on $\Gamma_{1}$. From our assumption there is the coordinates transformation $\Phi$ in the definition of property $(A)$.

We put

$$
h_{i}(x)=\left|D_{x} \xi_{i}\right|^{2}, \quad i=1, \ldots, n .
$$

and

$$
J(\xi)=\frac{\partial\left(x_{1}, \ldots, x_{n}\right)}{\partial\left(\xi_{1}, \ldots, \xi_{n}\right)} .
$$

Then $h_{i}>0$ in $B_{\delta}(P)$ and $J=\left(h_{1} \cdots h_{n}\right)^{-1 / 2}$. Obviously

$$
D_{x} u \cdot D_{x} v=h_{i} D_{\xi_{i}} u \cdot D_{\xi_{i}} v
$$


So, $\left|D_{x} u\right|^{2}=h_{i}\left(D_{\xi_{i}} u\right)^{2}$, which is written by $|E u|^{2}$. The first equation in (2.1) becomes

$$
\begin{aligned}
\varepsilon_{j} \int_{\Omega_{j}} D_{x} u_{j} \cdot D_{x} \varphi d x+\int_{\Omega_{j}} \frac{D_{x} u_{j} \cdot D_{x} \varphi}{\sqrt{1+\left|D_{x} u_{j}\right|^{2}}} d x=-n \int_{\Omega_{j}} H \varphi d x, & \\
\varphi & \in C_{0}^{\infty}\left(\Omega_{j}\right),
\end{aligned}
$$

from which we have

$$
\varepsilon_{j} D_{\xi_{i}}\left(J h_{i} D_{\xi_{i}} u_{j}\right)+D_{\xi_{i}}\left(\frac{J h_{i}}{\sqrt{1+\left|E u_{j}\right|^{2}}} D_{\xi_{i}} u_{j}\right)=n H J .
$$

We recall that $P$ is mapped to the origin in $\left(\xi_{1}, \ldots, \xi_{n}\right)$-space. From now on we consider $u_{j}$ only for sufficiently large $j$.

Our first object in this Section is to prove the following

Proposition 4.1. There is a positive number $\rho$ such that

$$
\begin{aligned}
\int_{B_{\rho}(O) \cap\left\{\xi_{n} \geqq 0\right\}}\left(\varepsilon_{j}+\frac{1}{\left(1+\left|D_{\xi} u_{j}\right|^{2}\right)^{3 / 2}}\right)\left|D_{\xi} D_{\xi_{k}} u_{j}\right|^{2} d \xi \leqq C(<\infty), \\
k=1, \ldots, n-1,
\end{aligned}
$$

where $\rho$ and $C$ are independent of $j$.

Proof. For simplicity $D_{\xi}, D_{\xi_{i}}, \ldots$ are denoted by $D, D_{i}, \ldots$, respectively. In the defnition of property (A) we take $\rho>0$ in such a way that $\Phi\left(B_{\delta}(P)\right) \supset B_{2 \rho}(O)$. Let $\zeta$ be a non-negative function in $C_{0}^{\infty}\left(B_{2 \rho}(O)\right)$. It may be assumed that $\zeta^{-1}|D \zeta|^{2}$ and $|D(|D \zeta|)|$ are bounded. For simplicity we denote $u_{j}$ and $\phi_{j}$ by $u$ and $\phi$, respectively. And we denote by $(, \quad)$ the $L^{2}\left(\left\{\xi_{n} \geqq 0\right\}\right)$-inner product. Let $k$ be any fixed integer such as $k=1, \ldots, n-1$. Setting $v=u-\phi\left(=u_{j}-\phi_{j}\right)$, we multiply (4.1) with $D_{k}\left(\zeta D_{k} v\right)$. Then

$$
\begin{aligned}
& \varepsilon_{j}\left(D_{i}\left(J h_{i} D_{i} u\right), D_{k}\left(\zeta D_{k} v\right)\right)+\left(D_{i}\left(\frac{J h_{i} D_{i} u}{\sqrt{1+|E u|^{2}}}\right), D_{k}\left(\zeta D_{k} v\right)\right) \\
& \quad=n\left(J H, D_{k}\left(\zeta D_{k} v\right)\right)
\end{aligned}
$$

We estimate the first term on the left-hand side of (4.2). By integration by parts

$$
\begin{aligned}
\left(D_{i}\left(J h_{i} D_{i} u\right), D_{k}\left(\zeta D_{k} v\right)\right) & =-\left(D_{i} D_{k}\left(J h_{i} D_{i} u\right), \zeta D_{k} v\right) \\
& =\left(D_{k}\left(J h_{i} D_{i} u\right), D_{i}\left(\zeta D_{k} v\right)\right)
\end{aligned}
$$


since $D_{k} v=0$ on $\left\{\xi_{n}=0\right\}$. This calculation needs that $u$ is in $C^{3}$. But it is avoided, because we can take an approximating sequence of $C^{3}$ functions for $u$. Hence

$$
\begin{aligned}
& \left(D_{i}\left(J h_{i} D_{i} u\right), D_{k}\left(\zeta D_{k} v\right)\right) \\
& \quad=\left(D_{k}\left(J h_{i} D_{i} v\right), D_{i}\left(\zeta D_{k} v\right)\right)+\left(D_{k}\left(J h_{i} D_{i} \phi\right), D_{i}\left(\zeta D_{k} v\right)\right) \\
& \quad \equiv I_{1}+I_{2}, \quad \text { say. }
\end{aligned}
$$

Obviously

$$
\begin{aligned}
I_{1}=( & \left.\zeta J h_{i},\left(D_{i} D_{k} v\right)^{2}\right)+\left(J h_{i} D_{i} \zeta, D_{k} v \cdot D_{i} D_{k} v\right) \\
& +\left(\zeta D_{k}\left(J h_{i}\right), D_{i} v \cdot D_{i} D_{k} v\right)+\left(D_{i} \zeta \cdot D_{k}\left(J h_{i}\right), D_{i} v \cdot D_{k} v\right) .
\end{aligned}
$$

From now on we denote by the same $C$ any positive constant independent of $j$. By Cauchy's inequality we have for $\delta>0$

$$
\left|\left(J h_{i} D_{i} \zeta, D_{k} v \cdot D_{i} D_{k} v\right)\right| \leqq \delta\left(\zeta J h_{i},\left(D_{i} D_{k} v\right)^{2}\right)+C(\delta)\left(\zeta^{-1}\left(D_{i} \zeta\right)^{2},\left(D_{k} v\right)^{2}\right)
$$

and

$$
\left|\left(\zeta D_{k}\left(J h_{i}\right), D_{i} v \cdot D_{i} D_{k} v\right)\right| \leqq \delta\left(\zeta J h_{i},\left(D_{i} D_{k} v\right)^{2}\right)+C(\delta)\left(\zeta,\left(D_{i} v\right)^{2}\right),
$$

where $C(\delta)$ depends on $\delta$ but not on $j$. Hence we obtain

$$
\begin{aligned}
I_{1} \geqq(1 & -2 \delta)\left(\zeta J h_{i},\left(D_{i} D_{k} v\right)^{2}\right) \\
& \quad-C(\delta)\left(\zeta+|D \zeta|+\zeta^{-1}|D \zeta|^{2},|D v|^{2}\right) .
\end{aligned}
$$

Next we write

$$
I_{2}=\left(\zeta D_{k}\left(J h_{i} D_{i} \phi\right), D_{i} D_{k} v\right)+\left(D_{i} \zeta \cdot D_{k}\left(J h_{i} D_{i} \phi\right), D_{k} v\right) .
$$

Let $M$ be a positive constant such that

$$
|\phi|,|D \phi|,\left|D D_{i} \phi\right| \leqq M
$$

where $M$ depends on the support of $\zeta$, but not on $j$. Then

$$
\left|\left(\zeta D_{k}\left(J h_{i} D_{i} \phi\right), D_{i} D_{k} v\right)\right| \leqq \delta\left(\zeta J h_{i},\left(D_{i} D_{k} v\right)^{2}\right)+C(\delta) M^{2},
$$

and

$$
\left|\left(D_{i} \zeta \cdot D_{k}\left(J h_{i} D_{i} \phi\right), D_{k} v\right)\right| \leqq C\left[\left(|D \zeta|,|D v|^{2}\right)+M^{2}\right]
$$


Hence

$$
I_{2} \geqq-\delta\left(\zeta J h_{i},\left(D_{i} D_{k} v\right)^{2}\right)-C(\delta)\left[\left(|D \zeta|,|D v|^{2}\right)+M^{2}\right] .
$$

Combining (4.4) and (4.5) with (4.3), we obtain

$$
\begin{aligned}
& \left(D_{i}\left(J h_{i} D_{i} u\right), D_{k}\left(\zeta D_{k} v\right)\right) \\
& \quad \geqq \frac{1}{2}\left(\zeta J h_{i},\left(D_{i} D_{k} v\right)^{2}\right)-C\left[\left(\zeta+|D \zeta|+\zeta^{-1}|D \zeta|^{2},|D v|^{2}\right)+M^{2}\right] .
\end{aligned}
$$

Here we note the following: If (4.3) is correct for $k=n$, then (4.6) is so.

Next we estimate the second term on the left-hand side of (4.2). Similarly by integration by parts

$$
\begin{aligned}
(4.7)( & \left.D_{i}\left(\frac{J h_{i} D_{i} u}{\sqrt{1+|E u|^{2}}}\right), D_{k}\left(\zeta D_{k} v\right)\right) \\
& =\left(D_{k}\left(\frac{J h_{i} D_{i} u}{\sqrt{1+|E u|^{2}}}\right), D_{i}\left(\zeta D_{k} v\right)\right) \\
& =\left(D_{k}\left(\frac{J h_{i} D_{i} u}{\sqrt{1+|E u|^{2}}}\right), D_{i}\left(\zeta D_{k} u\right)\right)-\left(D_{k}\left(\frac{J h_{i} D_{i} u}{\sqrt{1+|E u|^{2}}}\right), D_{i}\left(\zeta D_{k} \phi\right)\right) \\
& \equiv I_{3}+I_{4}, \quad \text { say. }
\end{aligned}
$$

If (4.7) holds for $k=n$, the following argument is also correct for the case of $k=n$, except for the terms $I_{32}$ and $K_{1}$. First we estimate $I_{3}$. Using the equality

$$
\begin{aligned}
D_{k}\left(\frac{J h_{i} D_{i} u}{\sqrt{1+|E u|^{2}}}\right)= & \frac{J h_{i}}{\sqrt{1+|E u|^{2}}}\left(D_{i} D_{k} u-\frac{h_{l} D_{l} u \cdot D_{i} u \cdot D_{k} D_{l} u}{1+|E u|^{2}}\right) \\
& +\frac{D_{k}\left(J h_{i}\right) \cdot D_{i} u}{\sqrt{1+|E u|^{2}}}-\frac{1}{2} \cdot \frac{D_{k} h_{l} \cdot\left(D_{l} u\right)^{2}}{\left(1+|E u|^{2}\right)^{3 / 2}} J h_{i} D_{i} u
\end{aligned}
$$

we have

$$
\begin{aligned}
I_{3}=( & \left.\frac{J \zeta h_{i}}{\sqrt{1+|E u|^{2}}},\left(D_{i} D_{k} u\right)^{2}-\frac{h_{l} D_{l} u \cdot D_{i} u \cdot D_{k} D_{l} u}{1+|E u|^{2}} D_{i} D_{k} u\right) \\
& +\left(\frac{\zeta D_{k}\left(J h_{i}\right)}{\sqrt{1+|E u|^{2}}}, D_{i} u \cdot D_{i} D_{k} u\right) \\
& -\frac{1}{2}\left(\frac{J \zeta h_{i} D_{k} h_{l}}{\left(1+|E u|^{2}\right)^{3 / 2}},\left(D_{l} u\right)^{2} D_{i} u \cdot D_{i} D_{k} u\right)
\end{aligned}
$$




$$
\begin{aligned}
& +\left(\frac{J h_{i} D_{i} \zeta}{\sqrt{1+|E u|^{2}}}, D_{i} D_{k} u \cdot D_{k} u-\frac{h_{l} D_{l} u \cdot D_{i} u \cdot D_{k} D_{l} u}{1+|E u|^{2}} D_{k} u\right) \\
& +\left(\frac{D_{k}\left(J h_{i}\right) \cdot D_{i} \zeta}{\sqrt{1+|E u|^{2}}}, D_{i} u \cdot D_{k} u\right) \\
& \quad-\frac{1}{2}\left(\frac{J h_{i} D_{i} \zeta \cdot D_{k} h_{l}}{\left(1+|E u|^{2}\right)^{3 / 2}},\left(D_{l} u\right)^{2} D_{i} u \cdot D_{k} u\right) \\
& \equiv \sum_{i=1}^{6} I_{3 i}, \quad \text { say. }
\end{aligned}
$$

Obviously

$$
\left|I_{35}\right|,\left|I_{36}\right| \leqq C(|D \zeta|,|D u|)
$$

We estimate $I_{34}$. The idea of the estimation of $I_{34}$ is due to [10]. Let us set three vectors as follows:

$$
\boldsymbol{a}=\left(\sqrt{h_{i}} D_{i} \zeta\right), \quad \boldsymbol{b}=\left(\sqrt{h_{i}} D_{i} u\right), \quad \boldsymbol{c}=\left(\sqrt{h_{i}} D_{i} D_{k} u\right)
$$

Then

$$
\begin{aligned}
& \left|h_{i} D_{i} \zeta \cdot D_{i} D_{k} u-\frac{h_{i} D_{i} \zeta \cdot h_{l} D_{l} u \cdot D_{i} u \cdot D_{k} D_{l} u}{1+|E u|^{2}}\right| \\
& \quad=\left|\boldsymbol{a} \cdot \boldsymbol{c}-\frac{(\boldsymbol{a} \cdot \boldsymbol{b})(\boldsymbol{b} \cdot \boldsymbol{c})}{1+|\boldsymbol{b}|^{2}}\right| \\
& \quad \leqq\left(|\boldsymbol{a}|^{2}-\frac{(\boldsymbol{a} \cdot \boldsymbol{b})^{2}}{1+|\boldsymbol{b}|^{2}}\right)^{1 / 2}\left(|\boldsymbol{c}|^{2}-\frac{(\boldsymbol{b} \cdot \boldsymbol{c})^{2}}{1+|\boldsymbol{b}|^{2}}\right)^{1 / 2}
\end{aligned}
$$

Hence we see that

$$
\left|I_{34}\right| \leqq\left(\frac{J|\boldsymbol{a}|\left|D_{k} u\right|}{\sqrt{1+|E u|^{2}}},\left(|\boldsymbol{c}|^{2}-\frac{(\boldsymbol{b} \cdot \boldsymbol{c})^{2}}{1+|\boldsymbol{b}|^{2}}\right)^{1 / 2}\right) .
$$

By Cauchy's inequality

$$
\begin{aligned}
\left|I_{34}\right| \leqq & \delta\left(\frac{J \zeta}{\sqrt{1+|E u|^{2}}}, h_{i}\left(D_{i} D_{k} u\right)^{2}-\frac{h_{i} h_{l} D_{i} u \cdot D_{l} u \cdot D_{i} D_{k} u \cdot D_{k} D_{l} u}{1+|E u|^{2}}\right) \\
& +C(\delta)\left(\zeta^{-1}|D \zeta|^{2},|D u|\right), \quad \delta>0 .
\end{aligned}
$$

Hence

$$
I_{31}+I_{34} \geqq \frac{1}{2} I_{31}-C\left(\zeta^{-1}|D \zeta|^{2},|D u|\right) .
$$


On the other hand

$$
\begin{aligned}
& \left(1+|E u|^{2}\right) h_{i}\left(D_{i} D_{k} u\right)^{2}-h_{i} h_{l} D_{i} u \cdot D_{l} u \cdot D_{i} D_{k} u \cdot D_{l} D_{k} u \\
& \quad=|\boldsymbol{c}|^{2}+|\boldsymbol{b}|^{2}|\boldsymbol{c}|^{2}-(\boldsymbol{b} \cdot \boldsymbol{c})^{2} \geqq|\boldsymbol{c}|^{2}
\end{aligned}
$$

Combining the above inequalities with (4.8) we obtain

$$
\begin{aligned}
I_{3} \geqq \frac{1}{2} & \left(\frac{J \zeta h_{i}}{\left(1+|E u|^{2}\right)^{3 / 2}},\left(D_{i} D_{k} u\right)^{2}\right)+I_{32}+I_{33} \\
& -C\left(|D \zeta|+\zeta^{-1}|D \zeta|^{2},|D u|\right) .
\end{aligned}
$$

Now we estimate the remained terms $I_{32}$ and $I_{33}$. By integration by parts

$$
I_{32}=-\frac{1}{2}\left(D_{k}\left(\frac{\zeta D_{k}\left(J h_{i}\right)}{\sqrt{1+|E u|^{2}}}\right),\left(D_{i} u\right)^{2}\right)
$$

Hence

$$
\begin{aligned}
& I_{32}=- \frac{1}{2}\left(\frac{D_{k}\left(\zeta D_{k}\left(J h_{i}\right)\right)}{\sqrt{1+|E u|^{2}}},\left(D_{i} u\right)^{2}\right) \\
&+\frac{1}{2}\left(\frac{\zeta D_{k}\left(J h_{i}\right)}{\left(1+|E u|^{2}\right)^{3 / 2}}, h_{l} D_{l} u \cdot D_{k} D_{l} u \cdot\left(D_{i} u\right)^{2}\right) \\
&+\frac{1}{4}\left(\frac{\zeta D_{k}\left(J h_{i}\right)}{\left(1+|E u|^{2}\right)^{3 / 2}}, D_{k} h_{l} \cdot\left(D_{l} u\right)^{2}\left(D_{i} u\right)^{2}\right) \\
& \equiv \sum_{i=1}^{3} J_{i}, \quad \text { say. }
\end{aligned}
$$

Easily

$$
\left|J_{1}\right|,\left|J_{3}\right| \leqq C(\zeta+|D \zeta|,|D u|)
$$

Since

$$
\begin{aligned}
J_{2}=\frac{1}{2} & \left(\frac{\zeta J D_{k} h_{i}}{\left(1+|E u|^{2}\right)^{3 / 2}}, h_{l} D_{l} u \cdot D_{k} D_{l} u \cdot\left(D_{i} u\right)^{2}\right) \\
& +\frac{1}{2}\left(\frac{\zeta D_{k} J \cdot h_{i}}{\left(1+|E u|^{2}\right)^{3 / 2}}, h_{l} D_{l} u \cdot D_{k} D_{l} u \cdot\left(D_{i} u\right)^{2}\right),
\end{aligned}
$$


we have

$$
\begin{aligned}
J_{2}+I_{33}= & \frac{1}{2}\left(\frac{\zeta D_{k} J \cdot h_{i}}{\left(1+|E u|^{2}\right)^{3 / 2}}, h_{l} D_{l} u \cdot D_{k} D_{l} u \cdot\left(D_{i} u\right)^{2}\right) \\
= & \frac{1}{2}\left(\frac{\zeta D_{k} J}{\left(1+|E u|^{2}\right)^{3 / 2}},|E u|^{2} h_{l} D_{l} u \cdot D_{k} D_{l} u\right) \\
= & \frac{1}{2}\left(\frac{\zeta D_{k} J}{\sqrt{1+|E u|^{2}}}, h_{l} D_{l} u \cdot D_{k} D_{l} u\right) \\
& -\frac{1}{2}\left(\frac{\zeta D_{k} J}{\left(1+|E u|^{2}\right)^{3 / 2}}, h_{l} D_{l} u \cdot D_{k} D_{l} u\right) .
\end{aligned}
$$

Setting

$$
K_{1}=\frac{1}{2}\left(\frac{\zeta D_{k} J}{\sqrt{1+|E u|^{2}}}, h_{l} D_{l} u \cdot D_{k} D_{l} u\right)
$$

we see that

$$
\left|J_{2}+I_{33}-K_{1}\right| \leqq C\left(\frac{\zeta}{\sqrt{1+|E u|^{2}}},\left|D D_{k} u\right|\right)
$$

By integration by parts

$$
K_{1}=-\frac{1}{4}\left(D_{k}\left(\frac{\zeta D_{k} J \cdot h_{l}}{\sqrt{1+|E u|^{2}}}\right),\left(D_{l} u\right)^{2}\right) .
$$

Hence

$$
\begin{aligned}
K_{1}=- & \frac{1}{4}\left(\frac{D_{k}\left(\zeta D_{k} J \cdot h_{l}\right)}{\sqrt{1+|E u|^{2}}},\left(D_{l} u\right)^{2}\right) \\
& +\frac{1}{4}\left(\frac{\zeta D_{k} J \cdot h_{l}}{\left(1+|E u|^{2}\right)^{3 / 2}}, h_{i} D_{i} u \cdot D_{i} D_{k} u \cdot\left(D_{l} u\right)^{2}\right) \\
& +\frac{1}{8}\left(\frac{\zeta D_{k} J \cdot D_{k} h_{i} \cdot h_{l}}{\left(1+|E u|^{2}\right)^{3 / 2}},\left(D_{i} u\right)^{2}\left(D_{l} u\right)^{2}\right) .
\end{aligned}
$$

Here we write with $K_{2}$ the second term on the right-hand side. Then

$$
K_{2}=\frac{1}{4}\left(\frac{\zeta D_{k} J}{\left(1+|E u|^{2}\right)^{3 / 2}},|E u|^{2} h_{i} D_{i} u \cdot D_{i} D_{k} u\right)
$$




$$
\begin{aligned}
& =\frac{1}{4}\left(\frac{\zeta D_{k} J}{\sqrt{1+|E u|^{2}}}, h_{i} D_{i} u \cdot D_{i} D_{k} u\right) \\
& \quad-\frac{1}{4}\left(\frac{\zeta D_{k} J}{\left(1+|E u|^{2}\right)^{3 / 2}}, h_{i} D_{i} u \cdot D_{i} D_{k} u\right) \\
& =\frac{1}{2} K_{1}-\frac{1}{4}\left(\frac{\zeta D_{k} J}{\left(1+|E u|^{2}\right)^{3 / 2}}, h_{i} D_{i} u \cdot D_{i} D_{k} u\right) .
\end{aligned}
$$

From the above we have

$$
\begin{aligned}
\left|K_{1}-K_{2}\right| & \leqq C(\zeta+|D \zeta|,|D u|), \\
\left|K_{2}-\frac{1}{2} K_{1}\right| & \leqq C\left(\frac{\zeta}{\sqrt{1+|E u|^{2}}},\left|D D_{k} u\right|\right) .
\end{aligned}
$$

Writing $\frac{1}{2} K_{1}=\left(K_{1}-K_{2}\right)+\left(K_{2}-\frac{1}{2} K_{1}\right)$, we obtain from these inequalities

$$
\left|K_{1}\right| \leqq C\left[\left(\frac{\zeta}{\sqrt{1+|E u|^{2}}},\left|D D_{k} u\right|\right)+(\zeta+|D \zeta|,|D u|)\right] .
$$

Therefore it follows from (4.12) that

$$
\left|J_{2}+I_{33}\right| \leqq C\left[\left(\frac{\zeta}{\sqrt{1+|E u|^{2}}},\left|D D_{k} u\right|\right)+(\zeta+|D \zeta|,|D u|)\right]
$$

Combining (4.10), (4.11) and (4.13) with (4.9), we conclude that

$$
\begin{aligned}
I_{3} \geqq & \frac{1}{2}\left(\frac{J \zeta h_{i}}{\left(1+|E u|^{2}\right)^{3 / 2}},\left(D_{i} D_{k} u\right)^{2}\right) \\
& -C\left[\left(\zeta+|D \zeta|+\zeta^{-1}|D \zeta|^{2},|D u|\right)+\left(\frac{\zeta}{\sqrt{1+|E u|^{2}}},\left|D D_{k} u\right|\right)\right]
\end{aligned}
$$

Lastly we estimate $I_{4}$. We can write

$$
\begin{aligned}
-I_{4}= & \left(\frac{J \zeta h_{i}}{\sqrt{1+|E u|^{2}}}, D_{i} D_{k} u \cdot D_{i} D_{k} \phi-\frac{h_{l} D_{l} u \cdot D_{i} u \cdot D_{k} D_{l} u}{1+|E u|^{2}} D_{i} D_{k} \phi\right) \\
& +\left(\frac{\zeta D_{k}\left(J h_{i}\right)}{\sqrt{1+|E u|^{2}}}, D_{i} u \cdot D_{i} D_{k} \phi\right)
\end{aligned}
$$




$$
\begin{aligned}
& -\frac{1}{2}\left(\frac{J \zeta h_{i} D_{k} h_{l}}{\left(1+|E u|^{2}\right)^{3 / 2}},\left(D_{l} u\right)^{2} D_{i} u \cdot D_{i} D_{k} \phi\right) \\
& +\left(\frac{J h_{i} D_{i} \zeta}{\sqrt{1+|E u|^{2}}}, D_{i} D_{k} u \cdot D_{k} \phi-\frac{h_{l} D_{l} u \cdot D_{i} u \cdot D_{k} D_{l} u}{1+|E u|^{2}} D_{k} \phi\right) \\
& +\left(\frac{D_{k}\left(J h_{i}\right) \cdot D_{i} \zeta}{\sqrt{1+|E u|^{2}}}, D_{k} \phi \cdot D_{i} u\right) \\
& -\frac{1}{2}\left(\frac{J h_{i} D_{i} \zeta \cdot D_{k} h_{l}}{\left(1+|E u|^{2}\right)^{3 / 2}}, D_{k} \phi \cdot\left(D_{l} u\right)^{2} D_{i} u\right)
\end{aligned}
$$

Hence

$$
\left|I_{4}\right| \leqq C M\left[1+\left(\frac{\zeta+|D \zeta|}{\sqrt{1+|E u|^{2}}},\left|D D_{k} u\right|\right)\right]
$$

Therefore, from (4.7) and (4.14), it follows that

$$
\begin{aligned}
& \left(D_{i}\left(\frac{J h_{i} D_{i} u}{\sqrt{1+|E u|^{2}}}\right), D_{k}\left(\zeta D_{k} v\right)\right) \geqq \frac{1}{2}\left(\frac{J \zeta h_{i}}{\left(1+|E u|^{2}\right)^{3 / 2}},\left(D_{i} D_{k} u\right)^{2}\right) \\
& -C(1+M)\left[1+\left(\zeta+|D \zeta|+\zeta^{-1}|D \zeta|^{2},|D u|\right)+\left(\frac{\zeta+|D \zeta|}{\sqrt{1+|E u|^{2}}},\left|D D_{k} u\right|\right)\right] .
\end{aligned}
$$

By Cauchy's inequality

$$
\begin{aligned}
\left(\frac{\zeta+|D \zeta|}{\sqrt{1+|E u|^{2}}},\left|D D_{k} u\right|\right) \leqq \delta & \left(\frac{J \zeta h_{i}}{\left(1+|E u|^{2}\right)^{3 / 2}},\left(D_{i} D_{k} u\right)^{2}\right) \\
& +C(\delta)\left(\zeta+\zeta^{-1}|D \zeta|^{2}, \sqrt{1+|E u|^{2}}\right)
\end{aligned}
$$

Accordingly we obtain

$$
\begin{aligned}
& \left(D_{i}\left(\frac{J h_{i} D_{i} u}{\sqrt{1+|E u|^{2}}}\right), D_{k}\left(\zeta D_{k} v\right)\right) \geqq \frac{1}{4}\left(\frac{J \zeta h_{i}}{\left(1+|E u|^{2}\right)^{3 / 2}},\left(D_{i} D_{k} u\right)^{2}\right) \\
& -C(M)\left[1+\left(\zeta+|D \zeta|+\zeta^{-1}|D \zeta|^{2},|D u|\right)\right]
\end{aligned}
$$

On the other hand

$$
\begin{aligned}
\left|\left(J H, D_{k}\left(\zeta D_{k} v\right)\right)\right| & =\left|\left(D_{k}(J H), \zeta D_{k} v\right)\right| \\
& \leqq C\|H\|_{1, \infty}(\zeta,|D v|) \\
& \leqq C\|H\|_{1, \infty}[(\zeta,|D u|)+M]
\end{aligned}
$$


where \|\|$_{1, \infty}$ is the $C^{0,1}(\bar{\Omega})$-norm. Combining the above, (4.6), (4.16) and (4.2) with (3.1), we finally conclude that

$$
\varepsilon_{j}\left(\zeta,\left|D D_{k} u\right|^{2}\right)+\left(\frac{\zeta}{\left(1+|E u|^{2}\right)^{3 / 2}},\left|D D_{k} u\right|^{2}\right)
$$

is uniformly bounded with respect to $j$. This completes the proof.

If we eliminate the assumption $k \neq n$ in Proposition 4.1, we have

Proposition 4.2. Let $\rho$ be the positive number in Proposition 4.1. Let $k=1, \ldots, n$. Then there is a positive constant $C$ independent of $j$ such that

$$
\int_{B_{\rho}(O) \cap\left\{\xi_{n} \geqq 0\right\}} \frac{\left|D_{\xi} D_{\xi_{k}} u_{j}\right|^{2}}{\left(1+\left|D_{\xi} u_{j}\right|^{2}\right)^{7 / 2}} d \xi \leqq C .
$$

Proof. The equation (4.1) can be written with

$$
J h_{i}\left(\varepsilon_{j} D_{i}^{2} u+D_{i}\left(\frac{D_{i} u}{\sqrt{1+|E u|^{2}}}\right)\right)=F_{1}+n J H
$$

where

$$
\left|F_{1}\right| \leqq C\left(1+\varepsilon_{j}|D u|\right)
$$

Hence

$$
\begin{aligned}
& J h_{n}\left(\varepsilon_{j} D_{n}^{2} u+D_{n}\left(\frac{D_{n} u}{\sqrt{1+|E u|^{2}}}\right)\right) \\
& =-\sum_{i \neq n} J h_{i}\left(\varepsilon_{j} D_{i}^{2} u+D_{i}\left(\frac{D_{i} u}{\sqrt{1+|E u|^{2}}}\right)\right)+F_{1}+n J H .
\end{aligned}
$$

This becomes

$$
\varepsilon_{j} D_{n}^{2} u+\frac{1}{\sqrt{1+|E u|^{2}}}\left(1-\frac{h_{n}\left(D_{n} u\right)^{2}}{1+|E u|^{2}}\right) D_{n}^{2} u=F_{2}+n h_{n}^{-1} H,
$$

where

$$
\left|F_{2}\right| \leqq C\left[1+\varepsilon_{j}|D u|+\left(\varepsilon_{j}+\frac{1}{\sqrt{1+|E u|^{2}}}\right) \sum_{(i, k) \neq(n, n)}\left|D_{i} D_{k} u\right|\right] .
$$


Accordingly

$$
\left(\varepsilon_{j}+\frac{1}{\left(1+|E u|^{2}\right)^{3 / 2}}\right)\left|D_{n}^{2} u\right| \leqq\left|F_{2}\right|+C|H|,
$$

which means that

$$
\begin{gathered}
\left(\frac{\zeta}{\left(1+|E u|^{2}\right)^{7 / 2}},\left(D_{n}^{2} u\right)^{2}\right) \\
\leqq C\left[1+\varepsilon_{j}^{2}(\zeta,|D u|)+\varepsilon_{j}^{2} \sum_{(i, k) \neq(n, n)}\left(\frac{\zeta}{\left(1+|E u|^{2}\right)^{1 / 2}},\left(D_{i} D_{k} u\right)^{2}\right)\right. \\
\left.+\sum_{(i, k) \neq(n, n)}\left(\frac{\zeta}{\left(1+|E u|^{2}\right)^{3 / 2}},\left(D_{i} D_{k} u\right)^{2}\right)\right] .
\end{gathered}
$$

Therefore from Proposition 4.1 and (3.1) we have obtained the required.

In Proposition 4.1, the estimation contains the second derivatives $D_{\xi} D_{\xi_{k}} u_{j}$, but we have assumed that $k \neq n$. If $k=n$, we have the following

Proposition 4.3. Suppose that $\partial u_{j} / \partial \mathbf{n} \geqq 0$ on $\Gamma_{1} \cap \partial \Omega_{j}$ for each $j$. Then there is a positive constant $d_{0}$ depending only on $\Gamma_{1}$ such that if $H \geqq$ $d_{0}$ on $\Gamma_{1}$, it holds that

$$
\int_{B_{\rho}(O) \cap\left\{\xi_{n} \geqq 0\right\}}\left(\varepsilon_{j}+\frac{1}{\left(1+\left|D_{\xi} u_{j}\right|^{2}\right)^{3 / 2}}\right)\left|D_{\xi} D_{\xi_{n}} u_{j}\right|^{2} d \xi \leqq C, \quad j=1,2, \ldots,
$$

where $\rho$ and $C$ are two positive constants independent of $j$.

Proof. As in the proof of Proposition 4.1, we denote $u_{j}\left(\phi_{j}\right)$ with $u(\phi)$, respectively. And $v=u-\phi\left(=u_{j}-\phi_{j}\right)$. Our assumption means that $D_{n} u \geqq 0$ on $\left\{\xi_{n}=0\right\}$. We write by $\langle$,$\rangle the L^{2}\left(\left\{\xi_{n}=0\right\}\right)$-inner product.

We multiply (4.1) with $D_{n}\left(\zeta D_{n} v\right)$, where $\zeta$ is the function in the proof of Proposition 4.1. We define

$$
I_{i}=\left\{\begin{array}{cl}
-\left\langle D_{i}\left(J h_{i} D_{i} u\right), \zeta D_{n} v\right\rangle & (i \neq n) \\
0 & (i=n) .
\end{array}\right.
$$


Then it holds that

$$
\left(D_{i}\left(J h_{i} D_{i} u\right), D_{n}\left(\zeta D_{n} v\right)=\left(D_{n}\left(J h_{i} D_{i} u\right), D_{i}\left(\zeta D_{n} v\right)\right)+I_{i},\right.
$$

In fact, it is trivial for $i=n$. If $i \neq n$, by integration by parts

$$
\begin{aligned}
& \left(D_{i}\left(J h_{i} D_{i} u\right), D_{n}\left(\zeta D_{n} v\right)\right) \\
& \quad=-\left\langle D_{i}\left(J h_{i} D_{i} u\right), \zeta D_{n} v\right\rangle-\left(D_{i} D_{n}\left(J h_{i} D_{i} u\right), \zeta D_{n} v\right) \\
& \quad=-\left\langle D_{i}\left(J h_{i} D_{i} u\right), \zeta D_{n} v\right\rangle+\left(D_{n}\left(J h_{i} D_{i} u\right), D_{i}\left(\zeta D_{n} v\right)\right) .
\end{aligned}
$$

Hence (4.17) is correct.

Similarly we obtain

$$
\begin{aligned}
& \left(D_{i}\left(\frac{J h_{i} D_{i} u}{\sqrt{1+|E u|^{2}}}\right), D_{n}\left(\zeta D_{n} v\right)\right) \\
& \quad=\left(D_{n}\left(\frac{J h_{i} D_{i} u}{\sqrt{1+|E u|^{2}}}\right), D_{i}\left(\zeta D_{n} v\right)\right)+J_{i}
\end{aligned}
$$

where

$$
J_{i}=\left\{\begin{array}{cc}
-\left\langle D_{i}\left(\frac{J h_{i} D_{i} u}{\sqrt{1+|E u|^{2}}}\right), \zeta D_{n} v\right\rangle & (i \neq n), \\
0 & (i=n) .
\end{array}\right.
$$

We estimate $I_{i}$ and $J_{i}$, respectively. Let $M$ be the constant in the proof of Proposition 4.1. First we see that

$$
\begin{aligned}
\left|I_{i}\right| & \leqq C M\left\langle\zeta,\left|D_{n} v\right|\right\rangle \\
& \leqq C M\left[\delta\left\langle\zeta,\left(D_{n} v\right)^{2}\right\rangle+C(\delta)\right], \quad \delta>0
\end{aligned}
$$

and

$$
\begin{aligned}
\left\langle\zeta,\left(D_{n} v\right)^{2}\right\rangle & =-\left(1, D_{n}\left(\zeta\left(D_{n} v\right)^{2}\right)\right) \\
& \leqq\left(\zeta+|D \zeta|,|D v|^{2}\right)+\left(\zeta,\left(D_{n}^{2} v\right)^{2}\right)
\end{aligned}
$$

Hence

$$
\left|I_{i}\right| \leqq C M\left[\delta\left(\zeta,\left(D_{n}^{2} v\right)^{2}\right)+\left(\zeta+|D \zeta|,|D v|^{2}\right)+C(\delta)\right]
$$

Next we estimate $J_{i}$ for $i \neq n$. By integration by parts

$$
\begin{aligned}
J_{i} & =\left\langle J h_{i} D_{i} u, \frac{D_{i}\left(\zeta D_{n} v\right)}{\sqrt{1+|E u|^{2}}}\right\rangle \\
& =\left\langle J h_{i} D_{i} u, \frac{D_{i}\left(\zeta D_{n} u\right)}{\sqrt{1+|E u|^{2}}}\right\rangle-\left\langle J h_{i} D_{i} u, \frac{D_{i}\left(\zeta D_{n} \phi\right)}{\sqrt{1+|E u|^{2}}}\right\rangle .
\end{aligned}
$$


Thus we can write

$$
J_{i}=A_{1}+A_{2}
$$

where

$$
A_{1}=\left\langle J h_{i} D_{i} \phi, \zeta \frac{D_{i} D_{n} u}{\sqrt{1+|E u|^{2}}}\right\rangle, \quad\left|A_{2}\right| \leqq C M .
$$

In general, let $f(t), g(t)$ and $h(t)$ be three given functions such that $g, h>0$. It is easily seen that

$$
\begin{aligned}
\left(\log \left(\sqrt{h} f+\sqrt{g+h f^{2}}\right)\right)^{\prime} \\
=\frac{1}{\sqrt{h} f+\sqrt{g+h f^{2}}} \\
\quad \times\left[\sqrt{h} f^{\prime}+(\sqrt{h})^{\prime} f+\frac{1}{2}\left(g+h f^{2}\right)^{-1 / 2}\left(2 h f f^{\prime}+h^{\prime} f^{2}+g^{\prime}\right)\right] \\
=\frac{\sqrt{h} f^{\prime}}{\sqrt{g+h f^{2}}}+\frac{(\sqrt{h})^{\prime} f}{\sqrt{h} f+\sqrt{g+h f^{2}}}+\frac{h^{\prime} f^{2}+g^{\prime}}{2 \sqrt{g+h f^{2}}\left(\sqrt{h} f+\sqrt{g+h f^{2}}\right)} .
\end{aligned}
$$

Hence

$$
\begin{gathered}
\frac{f^{\prime}}{\sqrt{g+h f^{2}}=}=\frac{1}{\sqrt{h}}\left(\log \left(\sqrt{h} f+\sqrt{g+h f^{2}}\right)\right)^{\prime}-\frac{h^{\prime}}{2 h} \frac{f}{\sqrt{h} f+\sqrt{g+h f^{2}}} \\
-\frac{1}{2 \sqrt{h}} \frac{h^{\prime} f^{2}+g^{\prime}}{\sqrt{g+h f^{2}}\left(\sqrt{h} f+\sqrt{g+h f^{2}}\right)} .
\end{gathered}
$$

We set

$$
f=D_{n} u, \psi=\sum_{k \neq n} h_{k}\left(D_{k} u\right)^{2}, g=1+\psi \text { and } h=h_{n} .
$$

From the above, it follows that

$$
\begin{aligned}
\frac{D_{i} D_{n} u}{\sqrt{1+|E u|^{2}}=\frac{1}{\sqrt{h_{n}}}} D_{i}\left(\log \left(\sqrt{h_{n}} D_{n} u+\sqrt{1+|E u|^{2}}\right)\right) \\
-\frac{1}{2 h_{n}} \frac{D_{i} h_{n} \cdot D_{n} u}{\sqrt{h_{n}} D_{n} u+\sqrt{1+|E u|^{2}}} \\
-\frac{1}{2 \sqrt{h_{n}}} \frac{D_{i} h_{n} \cdot\left(D_{n} u\right)^{2}}{\sqrt{1+|E u|^{2}}\left(\sqrt{h_{n}} D_{n} u+\sqrt{1+|E u|^{2}}\right)} \\
-\frac{1}{2 \sqrt{h_{n}}} \frac{D_{i} \psi}{\sqrt{1+|E u|^{2}}\left(\sqrt{h_{n}} D_{n} u+\sqrt{1+|E u|^{2}}\right)} .
\end{aligned}
$$


Thus we have

$$
\begin{aligned}
(4.21) A_{1}=\left\langle J h_{i} \frac{\zeta}{\sqrt{h_{n}}} D_{i} \phi, D_{i}\left(\log \left(\sqrt{h_{n}} D_{n} u+\sqrt{1+|E u|^{2}}\right)\right)\right\rangle \\
\quad-\frac{1}{2}\left\langle J h_{i} \frac{D_{i} h_{n}}{h_{n}} \zeta D_{i} \phi, \frac{D_{n} u}{\sqrt{h_{n}} D_{n} u+\sqrt{1+|E u|^{2}}}\right\rangle \\
\quad-\frac{1}{2}\left\langle J h_{i} \frac{D_{i} h_{n}}{\sqrt{h_{n}}} \zeta D_{i} \phi, \frac{\left(D_{n} u\right)^{2}}{\sqrt{1+|E u|^{2}}\left(\sqrt{h_{n}} D_{n} u+\sqrt{1+|E u|^{2}}\right)}\right\rangle \\
\quad-\frac{1}{2}\left\langle J h_{i} \frac{\zeta}{\sqrt{h_{n}}} D_{i} \phi, \frac{D_{i} \psi}{\sqrt{1+|E u|^{2}}\left(\sqrt{h_{n}} D_{n} u+\sqrt{1+|E u|^{2}}\right)}\right\rangle \\
\equiv \sum_{i=1}^{4} A_{1 i}, \quad \text { say. }
\end{aligned}
$$

Since $D_{n} u \geqq 0$ on $\left\{\xi_{n}=0\right\}$, we have

$$
1 \leqq \sqrt{h_{n}} D_{n} u+\sqrt{1+|E u|^{2}} \leqq 2 \sqrt{1+|E u|^{2}}
$$

from which

$$
0 \leqq \log \left(\sqrt{h_{n}} D_{n} u+\sqrt{1+|E u|^{2}}\right) \leqq \log 2+\frac{1}{2} \log \left(1+|E u|^{2}\right) \quad \text { on }\left\{\xi_{n}=0\right\}
$$

Now we estimate $A_{11}$. By integration by parts

$$
A_{11}=-\left\langle D_{i}\left(J h_{i} \frac{\zeta}{\sqrt{h_{n}}} D_{i} \phi\right), \log \left(\sqrt{h_{n}} D_{n} u+\sqrt{1+|E u|^{2}}\right)\right\rangle .
$$

Hence

$$
\left|A_{11}\right| \leqq C M\left[1+\left\langle\zeta+|D \zeta|, \log \left(1+|E u|^{2}\right)\right\rangle\right]
$$

Since

$$
\left\langle\zeta+|D \zeta|, \log \left(1+|E u|^{2}\right)\right\rangle=-\left(1, D_{n}\left((\zeta+|D \zeta|) \log \left(1+|E u|^{2}\right)\right)\right)
$$

and

$$
\left|D_{n} \log \left(1+|E u|^{2}\right)\right| \leqq C\left(1+\frac{\left|D D_{n} u\right|}{\sqrt{1+|E u|^{2}}}\right)
$$

we obtain

$$
\left|A_{11}\right| \leqq C M\left[1+\left(\zeta+|D \zeta|, \frac{\left|D D_{n} u\right|}{\sqrt{1+|E u|^{2}}}\right)+\left(|D \zeta|+|D| D \zeta||, \sqrt{1+|E u|^{2}}\right)\right]
$$


Obviously $\left|A_{12}\right|,\left|A_{13}\right| \leqq C M$, and

$$
\left|D_{i} \psi\right| \leqq C\left[\sum_{k \neq n}\left(D_{k} \phi\right)^{2}+\sum_{i, k \neq n}\left|D_{i} \phi\right|\left|D_{i} D_{k} \phi\right|\right] \quad \text { on }\left\{\xi_{n}=0\right\}
$$

Hence

$$
\left|A_{14}\right| \leqq C M
$$

From the above and (4.21) it follows that

$$
\begin{aligned}
&\left|A_{1}\right| \leqq C M {\left[1+\left(\zeta+|D \zeta|, \frac{\left|D D_{n} u\right|}{\sqrt{1+|E u|^{2}}}\right)\right.} \\
&\left.+\left(|D \zeta|+|D(|D \zeta|)|, \sqrt{1+|E u|^{2}}\right)\right] .
\end{aligned}
$$

Therefore we obtain by (4.20)

$$
\left|J_{i}\right| \leqq \text { the right-hand side of (4.22) }
$$

By Cauchy's inequality it follows that

$$
\begin{aligned}
\left|J_{i}\right| \leqq C M & {\left[1+\delta\left(\zeta, \frac{\left|D D_{n} u\right|^{2}}{\left(1+|E u|^{2}\right)^{3 / 2}}\right)\right.} \\
+ & \left.C(\delta)\left(\zeta+|D \zeta|+\zeta^{-1}|D \zeta|^{2}+|D(|D \zeta|)|, \sqrt{1+|E u|^{2}}\right)\right]
\end{aligned}
$$

Lastly we have

$$
\left(J H, D_{n}\left(\zeta D_{n} v\right)\right)=\left(J H, D_{n}\left(\zeta D_{n} u\right)\right)-\left(J H, D_{n}\left(\zeta D_{n} \phi\right)\right)
$$

and

$$
\left(J H, D_{n}\left(\zeta D_{n} u\right)\right)=-\left(D_{n}(J H), \zeta D_{n} u\right)-\left\langle J H, \zeta D_{n} u\right\rangle .
$$

Hence combining (4.17) and (4.18) with (4.1), we obtain

$$
\begin{aligned}
(4.24) \varepsilon_{j}\left(D_{n}\left(J h_{i} D_{i} u\right), D_{i}\left(\zeta D_{n} v\right)\right) \\
\quad+\left(D_{n}\left(\frac{J h_{i} D_{i} u}{\sqrt{1+|E u|^{2}}}\right), D_{i}\left(\zeta D_{n} v\right)\right)+n\left\langle J H, \zeta D_{n} u\right\rangle \\
=-n\left(D_{n}(J H), \zeta D_{n} u\right)-n\left(J H, D_{n}\left(\zeta D_{n} \phi\right)\right)-\varepsilon_{j} \sum_{i} I_{i}-\sum_{i} J_{i} .
\end{aligned}
$$


Here we use (4.19), (4.23) and (3.1). Then we can put the right hand side of $(4.24)=G$,

where

$$
|G| \leqq C(M+1)\left[\varepsilon_{j} \delta\left(\zeta,\left(D_{n}^{2} u\right)^{2}\right)+\varepsilon_{j} M^{2}+\delta\left(\zeta, \frac{\left|D D_{n} u\right|^{2}}{\left(1+|E u|^{2}\right)^{3 / 2}}\right)+C(\delta)\right] .
$$

Hereafter we proceed in parallel with the proof of Proposition 4.1, by replacing $D_{k}$ with $D_{n}$. The situation is different only for the two terms $I_{32}$ and $K_{1}$ in the proof of Proposition 4.1. By integration by parts

$$
I_{32}=-\frac{1}{2}\left(D_{n}\left(\frac{\zeta D_{n}\left(J h_{l}\right)}{\sqrt{1+|E u|^{2}}}\right),\left(D_{l} u\right)^{2}\right)-\frac{1}{2}\left\langle\frac{\zeta D_{n}\left(J h_{l}\right)}{\sqrt{1+|E u|^{2}}},\left(D_{l} u\right)^{2}\right\rangle
$$

and

$$
K_{1}=-\frac{1}{4}\left(D_{n}\left(\frac{\zeta D_{n} J \cdot h_{l}}{\sqrt{1+|E u|^{2}}}\right),\left(D_{l} u\right)^{2}\right)-\frac{1}{4}\left\langle\zeta \frac{D_{n} J \cdot h_{l}}{\sqrt{1+|E u|^{2}}},\left(D_{l} u\right)^{2}\right\rangle .
$$

On the last equality we set $K_{1}=\tilde{K}_{1}+L$. Let $K_{2}$ be the same in the proof of Proposition 4.1, where $k$ is replaced with $n$. Let $A_{i}(i=1,2, \ldots)$ be the terms, which will be decided later such that

$$
\left|A_{i}\right| \leqq C\left[\left(\zeta, \frac{\left|D D_{n} u\right|}{\sqrt{1+|E u|^{2}}}\right)+(\zeta+|D \zeta|,|D u|)\right]
$$

Then we can write

$$
K_{2}=\frac{1}{2}\left(\tilde{K}_{1}+L\right)-A_{1}, \quad J_{2}+I_{33}=\tilde{K}_{1}+L+A_{2}
$$

and

$$
\tilde{K}_{1}=K_{2}+A_{3}
$$

Here we use the equality:

$$
\begin{aligned}
\frac{1}{2} \tilde{K}_{1} & =\left(\tilde{K}_{1}-K_{2}\right)+\left(K_{2}-\frac{1}{2} \tilde{K}_{1}\right) \\
& =\frac{1}{2} L+A_{3}-A_{1} .
\end{aligned}
$$

Then

$$
J_{2}+I_{33}=2 L+\left(2 A_{3}-2 A_{1}+A_{2}\right)
$$


Thus (4.13) holds for $k=n$. It needs to estimate the above two boundary integrals. The remained part of the proof is similar to that of Proposition 4.1. If $l \neq n, D_{l} u=D_{l} \phi$ on $\left\{\xi_{n}=0\right\}$ and

$$
\left|\left\langle\frac{\zeta D_{n}\left(J h_{l}\right)}{\sqrt{1+|E u|^{2}}},\left(D_{l} \phi\right)^{2}\right\rangle\right|,\left|\left\langle\frac{\zeta D_{n} J \cdot h_{l}}{\sqrt{1+|E u|^{2}}},\left(D_{l} \phi\right)^{2}\right\rangle\right| \leqq C M .
$$

Hence, from (4.24) it is enough to prove

$$
\begin{gathered}
n\left\langle J H, \zeta D_{n} u\right\rangle-\frac{1}{2}\left\langle\frac{\zeta D_{n}\left(J h_{n}\right)}{\sqrt{1+|E u|^{2}}},\left(D_{n} u\right)^{2}\right\rangle \\
-\frac{1}{2}\left\langle\frac{\zeta D_{n} J \cdot h_{n}}{\sqrt{1+|E u|^{2}}},\left(D_{n} u\right)^{2}\right\rangle \geqq 0 .
\end{gathered}
$$

Since $D_{n} u \geqq 0$ on $\left\{\xi_{n}=0\right\}$, we have

$$
D_{n} u \geqq \sqrt{h_{n}} \frac{\left(D_{n} u\right)^{2}}{\sqrt{1+|E u|^{2}}},
$$

from which (4.26) holds if

$$
n J \sqrt{h_{n}} H-\frac{1}{2} D_{n}\left(J h_{n}\right)-\frac{1}{2} D_{n} J \cdot h_{n} \geqq 0 \quad \text { on }\left\{\xi_{n}=0\right\} .
$$

Thus we can take $d_{0}$ as follows:

$$
J d_{0} \geqq \frac{1}{2 n}\left(\frac{1}{\sqrt{h_{n}}} D_{n}\left(J h_{n}\right)+D_{n} J \cdot \sqrt{h_{n}}\right) .
$$

We have finished the proof.

We give an example of the constant $d_{0}$ in (4.27).

ExAMPLE 3. Let us denote by $C_{R}$ the circumference with its center $(0,-R)$ and with its radius $R$. Let $\Gamma_{1}$ be an open arc on $C_{R}$ such that $\Gamma_{1}$ contains the origin (see Figure 3 ). Let $\Omega$ be a bounded domain in the exterior of $C_{R}$ such that $\partial \Omega \supset \Gamma_{1}$. We transform $\Gamma_{1}$ into a flat and calculate $J$ and $h_{2}$. For this sake we use the polar coordinates transformation $x=\xi_{2} \cos \xi_{1}$, $y=-\xi_{2} \sin \xi_{1}-R$. Then

$$
J=\frac{D(x, y)}{D\left(\xi_{1}, \xi_{2}\right)}=\xi_{2}, \quad h_{2}=\left(D_{x} \xi_{2}\right)^{2}+\left(D_{y} \xi_{2}\right)^{2}=1
$$

Hence

$$
\text { the right-hand side of }(4.27)=\frac{1}{2} \text {, }
$$

namely we can take $d_{0}=1 / 2 R$. 


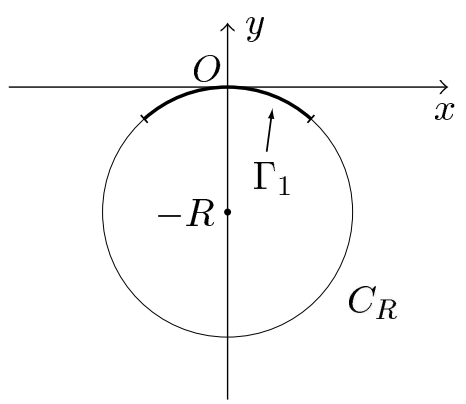

Figure 3.

\section{$\S 5$. Proof of our Theorems}

Let $\left\{u_{j}\right\}$ be the sequence of solutions in (2.1) and $u$ be the function in (2.2). We recall that (3.5) holds. We denote by the same $\{\nu\}$ any subsequence of $\{j\}$. Before proving our Theorems we prepare the following

Proposition 5.1. There is a positive sequence $\left\{\alpha_{\nu}\right\}$ with $\alpha_{\nu} \rightarrow 0$ $(\nu \rightarrow \infty)$ such that for $1 \leqq i \leqq n$

$$
\frac{D_{i} u_{\nu}}{\left(1+\left|D u_{\nu}\right|^{2}\right)^{\alpha_{\nu}}} \rightarrow D_{i} u \quad \text { in } L^{1}\left(\Omega^{\prime}\right) \text { as } \nu \rightarrow \infty
$$

where $\Omega^{\prime}$ is any subdomain of $\Omega$ such that $\partial \Omega^{\prime} \cap \partial \Omega \subset \Gamma_{1}$ and $\bar{\Omega}^{\prime} \cap \Gamma_{2}=\phi$.

Proof. By the convergence theorem

$$
\frac{D_{i} u}{\left(1+\left|D u_{\nu}\right|^{2}\right)^{\alpha_{\nu}}} \rightarrow D_{i} u \quad \text { in } L^{1}\left(\Omega^{\prime}\right)(\nu \rightarrow \infty) .
$$

Hence it is sufficient to prove that

$$
\frac{D_{i}\left(u_{\nu}-u\right)}{\left(1+\left|D u_{\nu}\right|^{2}\right)^{\alpha_{\nu}}} \rightarrow 0 \quad \text { in } L^{1}\left(\Omega^{\prime}\right)(\nu \rightarrow \infty)
$$

From (2.2) we can take a sequence $\left\{G_{k}\right\}$, subdomains of $\Omega$ such that $\bar{G}_{k} \subset \Omega, G_{k} \uparrow \Omega(k \rightarrow \infty)$ and

$$
D_{i} u_{\nu} \rightrightarrows D_{i} u \quad \text { in } G_{k}(\nu \rightarrow \infty) .
$$


We denote with the same notation any subsequence of $\left\{u_{\nu}\right\}$. Then we may assume that

$$
\left|D_{i} u_{\nu}-D_{i} u\right|<\frac{1}{\nu} \quad \text { in } G_{\nu}
$$

Let us take a positive sequence $\left\{\alpha_{\nu}\right\}$ such that $\alpha_{\nu} \rightarrow 0(\nu \rightarrow \infty)$ and

$$
\left|\Omega-G_{\nu}\right|^{2 \alpha_{\nu}} \rightarrow 0 \quad(\nu \rightarrow \infty)
$$

We set

$$
\int_{\Omega^{\prime}} \frac{\left|D_{i}\left(u_{\nu}-u\right)\right|}{\left(1+\left|D u_{\nu}\right|^{2}\right)^{\alpha_{\nu}}} d x=\int_{G_{\nu}}+\int_{\Omega^{\prime}-G_{\nu}} \equiv I_{\nu}+J_{\nu}, \quad \text { say. }
$$

Easily, $I_{\nu} \rightarrow 0(\nu \rightarrow \infty)$. And

$$
\left|J_{\nu}\right| \leqq \int_{\Omega^{\prime}-G_{\nu}}\left|D_{i} u_{\nu}\right|^{1-2 \alpha_{\nu}} d x+\int_{\Omega^{\prime}-G_{\nu}}\left|D_{i} u\right| d x .
$$

Since $u \in W^{1,1}(\Omega)$,

$$
\int_{\Omega-G_{\nu}}\left|D_{i} u\right| d x \rightarrow 0 \quad(\nu \rightarrow \infty) .
$$

By Hölder's inequality

$$
\int_{\Omega^{\prime}-G_{\nu}}\left|D_{i} u_{\nu}\right|^{1-2 \alpha_{\nu}} d x \leqq\left|\Omega-G_{\nu}\right|^{2 \alpha_{\nu}}\left(\int_{\Omega^{\prime}}\left|D u_{\nu}\right| d x\right)^{1-2 \alpha_{\nu}} .
$$

Hence $J_{\nu} \rightarrow 0(\nu \rightarrow \infty)$ from (5.2) and (3.1). Thus we obtain (5.1). This completes the proof.

Now we prove Theorem 1.

Proof of Theorem 1. Let $P$ be any fixed point on $\Gamma_{1}$ and $\delta$ be a sufficiently small positive number. Let $\zeta$ be any fixed function in $C_{0}^{\infty}\left(B_{\delta}(P)\right)$. Since from $(3.3)$

$$
\left|D_{i}\left(\frac{u_{\nu}-\phi_{\nu}}{\left(1+\left|D u_{\nu}\right|^{2}\right)^{5 / 4}}\right)\right| \leqq C\left(1+\frac{\left|D D_{i} u_{\nu}\right|}{\left(1+\left|D u_{\nu}\right|^{2}\right)^{7 / 4}}\right) \quad \text { in } B_{\delta}(P),
$$

it follows from Proposition 4.2 that $\left\{\zeta\left(u_{\nu}-\phi_{\nu}\right) /\left(1+\left|D u_{\nu}\right|^{2}\right)^{5 / 4}\right\}$ is uniformly bounded in $W_{0}^{1,2}(\Omega)$. Hence a subsequence of $\left\{\zeta\left(u_{\nu}-\phi_{\nu}\right) /\left(1+\left|D u_{\nu}\right|^{2}\right)^{5 / 4}\right\}$ 
converges weakly to a function $w$ in $W_{0}^{1,2}(\Omega)$. On the other hand, from $(2.2)$ it converges to $\left\{\zeta(u-\phi) /\left(1+|D u|^{2}\right)^{5 / 4}\right\}$ pointwise in $\Omega$. Therefore by the usual argument

$$
w=\frac{\zeta(u-\phi)}{\left(1+|D u|^{2}\right)^{5 / 4}} \in W_{0}^{1,2}(\Omega),
$$

which implies that $(u-\phi) /\left(1+|D u|^{2}\right)^{5 / 4} \in W_{0}^{1,2}\left(\Omega ; \Gamma_{1}\right)$. In Section 2 we have already stated that the boundary condition is satisfied on $\Gamma_{2}$. Thus Theorem 1 holds.

Proof of Theorem 2. We take the sequence $\left\{\alpha_{\nu}\right\}$ in Proposition 5.1. Let $1 \leqq i \leqq n$. We have

$$
D_{i}\left(\frac{u_{\nu}-\phi_{\nu}}{\left(1+\left|D u_{\nu}\right|^{2}\right)^{\alpha_{\nu}}}\right)-\frac{D_{i}\left(u_{\nu}-\phi_{\nu}\right)}{\left(1+\left|D u_{\nu}\right|^{2}\right)^{\alpha_{\nu}}}=-2 \alpha_{\nu}\left(u_{\nu}-\phi_{\nu}\right) \frac{D u_{\nu} \cdot D D_{i} u_{\nu}}{\left(1+\left|D u_{\nu}\right|^{2}\right)^{\alpha_{\nu}+1}} .
$$

Hence from (3.3)

$$
\left|D_{i}\left(\frac{u_{\nu}-\phi_{\nu}}{\left(1+\left|D u_{\nu}\right|^{2}\right)^{\alpha_{\nu}}}\right)-\frac{D_{i}\left(u_{\nu}-\phi_{\nu}\right)}{\left(1+\left|D u_{\nu}\right|^{2}\right)^{\alpha_{\nu}}}\right| \leqq C \alpha_{\nu} \frac{\left|D D_{i} u_{\nu}\right|}{\left(1+\left|D u_{\nu}\right|^{2}\right)^{\left(2 \alpha_{\nu}+1\right) / 2}} .
$$

Let $P$ be any fixed point in $\Gamma_{1}$ and $\delta>0$ be small. Then from Proposition 4.3

$$
\int_{B_{\delta}(P) \cap \Omega_{\nu}} \frac{\left|D D_{i} u_{\nu}\right|^{2}}{\left(1+\left|D u_{\nu}\right|^{2}\right)^{3 / 2}} d x \leqq C
$$

Accordingly by Schwarz inequality

$$
\begin{aligned}
& \int_{B_{\delta}(P) \cap \Omega_{\nu}} \frac{\left|D D_{i} u_{\nu}\right|}{\left(1+\left|D u_{\nu}\right|^{2}\right)^{\left(2 \alpha_{\nu}+1\right) / 2}} d x \\
& \quad \leqq\left(\int_{\Omega_{\nu}} \sqrt{1+\left|D u_{\nu}\right|^{2}} d x\right)^{1 / 2}\left(\int_{B_{\delta}(P) \cap \Omega_{\nu}} \frac{\left|D D_{i} u_{\nu}\right|^{2}}{\left(1+\left|D u_{\nu}\right|^{2}\right)^{3 / 2}} d x\right)^{1 / 2} \\
& \quad \leqq C \quad(\text { from }(3.1)) .
\end{aligned}
$$

We take any function $\psi \in C_{0}^{\infty}\left(B_{\delta}(P)\right)$ and denote by $($,$) the L^{2}(\Omega)$ inner product. Then from the above it follows that

$$
\left(D_{i}\left(\frac{u_{\nu}-\phi_{\nu}}{\left(1+\left|D u_{\nu}\right|^{2}\right)^{\alpha_{\nu}}}\right), \psi\right)-\left(\frac{D_{i}\left(u_{\nu}-\phi_{\nu}\right)}{\left(1+\left|D u_{\nu}\right|^{2}\right)^{\alpha_{\nu}}}, \psi\right) \rightarrow 0 \quad(\nu \rightarrow \infty) .
$$


On the other hand

$$
\left(D_{i}\left(\frac{u_{\nu}-\phi_{\nu}}{\left(1+\left|D u_{\nu}\right|^{2}\right)^{\alpha_{\nu}}}\right), \psi\right)=-\left(\frac{u_{\nu}-\phi_{\nu}}{\left(1+\left|D u_{\nu}\right|^{2}\right)^{\alpha_{\nu}}}, D_{i} \psi\right)
$$

and

$$
\frac{D_{i} \phi_{\nu}}{\left(1+\left|D u_{\nu}\right|^{2}\right)^{\alpha_{\nu}}} \rightarrow D_{i} \phi \quad \text { in } L^{1}(\Omega \cap\{\operatorname{supp} \psi\})(\nu \rightarrow \infty)
$$

Hence from Proposition 5.1 we have

$$
\left(\frac{D_{i}\left(u_{\nu}-\phi_{\nu}\right)}{\left(1+\left|D u_{\nu}\right|^{2}\right)^{\alpha_{\nu}}}, \psi\right) \rightarrow\left(D_{i}(u-\phi), \psi\right) \quad(\nu \rightarrow \infty)
$$

Further it follows from (2.2), (3.3) and the convergence theorem that

$$
\frac{u_{\nu}-\phi_{\nu}}{\left(1+\left|D u_{\nu}\right|^{2}\right)^{\alpha_{\nu}}} \rightarrow u-\phi \quad \text { in } L^{1}(\Omega \cap\{\operatorname{supp} \psi\})(\nu \rightarrow \infty)
$$

Combining the above with (5.3), we obtain

$$
\left(u-\phi, D_{i} \psi\right)=-\left(D_{i}(u-\phi), \psi\right),
$$

which means that $u-\phi \in W_{0}^{1,1}\left(\Omega ; \Gamma_{1}\right)$.

Next it is known that $u \in C^{0}\left(\Omega \cup \Gamma_{2}\right)$ and $u=\phi$ on $\Gamma_{2}$, by the usual method of barrier functions (see e.g., [18]).

Lastly we have

$\left|D_{i}\left(\frac{u_{\nu}-\phi_{\nu}}{\left(1+\left|D u_{\nu}\right|^{2}\right)^{1 / 4}}\right)\right| \leqq C\left(\left(1+\left|D u_{\nu}\right|^{2}\right)^{1 / 4}+\frac{\left|D D_{i} u_{\nu}\right|}{\left(1+\left|D u_{\nu}\right|^{2}\right)^{3 / 4}}\right)$ in $B_{\delta}(P)$.

Using Proposition 4.3, we proceed in parallel with the proof of Theorem 1. Then the final statement in Theorem 2 is obtained. We complete the proof.

\section{REFERENCES}

[1] C. Gerhardt, Existence, regularity, and boundary behaviour of generalized surfaces of prescribed mean curvature, Math. Z., 139 (1974), 173-198.

[2] M. Giaquinta, On the Dirichlet problem for surfaces of prescribed mean curvature, Manuscripta Math., 12 (1974), 73-86.

[3] D. Gilberg and N. S. Trudinger, Elliptic Partial Differential Equations of Second Order, Springer, New York, 1983. 
[4] A. Giusti, Boundary value problems for non-parametric surfaces of prescribed mean curvature, Ann. Scuola Norm. Sup. Pisa Cl. Sci. (4), 3 (1976), 501-548.

[5] G. Gregori, Generalized solutions for a class of non-uniformly elliptic equations in divergence form, Comm. Partial Differential Equations, 22 (1997), 581-617.

[6] N. M. Ivochkina, The Dirichlet problem for the equations of curvature of order $m$, Leningrad Math. J., 2 (1991), 631-654.

[7] H. Jenkins and J. Serrin, The Dirichlet problem for the minimal surface equation in higher dimensions, J. Reine Angew. Math., 229 (1968), 170-187.

[8] Z. Jin and K. Lancaster, Behavior of solutions for some Dirichlet problems near reentrant corners, Indiana Univ. Math. J., 46 (1997), 827-862.

[9] N. J. Korevaar and L. Simon, Continuity estimates for solutions to the prescribedcurvature Dirichlet problem, Math. Z., 197 (1987), 457-464.

[10] O. A. Ladyzhenskaya and N. N. Ural'ceva, Local estimates for gradients of solutions of non-uniformly elliptic and parabolic equations, Comm. Pure Appl. Math., 23 (1970), 677-703.

[11] L L L Lal estimates of the gradients of solution to a simplest regularization for some class of non uniformly elliptic equations, Zap. Nauchn. Sem. S.-Peterburg Otdel Mat. Inst. Steklov, 213 (1994), 75-92, (Russian).

[12] K. E. Lancaster, Nonexistence of some nonparametric surfaces of prescribed mean curvature, Proc. Amer. Math. Soc., 96 (1986), 187-188.

[13] C. P. Lau and F. H. Lin, The best Hölder exponent for solutions of the non-parametric least area problem, Indiana Univ. Math. J., 34 (1985), 809-813.

[14] C. P. Lau, Quasilinear elliptic equations with small boundary data, Manuscripta Math., 53 (1985), 77-99.

[15] A. Lichnewsky, Sur le comportement au bord des solutions généralisées du problème non paramétrique des surfaces minimals, J. Math. Pures Appl., 53 (1974), 397-476.

[16] F. Schultz and G. H. Williams, Barriers and existence results for a class of equations of mean curvature type, Analysis, 7 (1987), 359-374.

[17] J. Serrin, The problem of Dirichlet for quasilinear elliptic differential equations with many independent variables, Phil. Trans. Royal Soc. London, A 264 (1969), 413-496.

[18] L. Simon, Interior gradient bounds for non-uniformly elliptic equations, Indiana Univ. Math. J., 25 (1976), 821-855.

[19] — Boundary behaviour of solutions of the non-parametric least area problem, Bull. Austral. Math. Soc., 26 (1982), 17-27.

[20] R. Teman, Applications de l'analyse convexe au calcul des variations, Lecture Notes in Math., 543, Springer, 1975.

[21] A. S. Tersenov, On quasilinear non-uniformly elliptic equations in some non-convex domains, Comm. Partial Differential Equations, 23 (1998), 2165-2185.

[22] G. H. Williams, The Dirichlet problem for the minimal surface equation with Lipschitz continuous boundary data, J. Reine Anegew. Math., 354 (1984), 123-140.

[23] Solutions of the minimal surface equation-continuous and discontinuous at the boundary, Comm. Partial Differential Equations, 11 (1986), 1439-1457. 
Kazuya Hayasida

Department of Computational Science

Faculty of Science

Kanazawa University

Kanazawa, 920-1192

Japan

Masao Nakatani

Kanazawa Institute of Technology

Kanazawa, 921-8501

Japan 